\title{
Remediation of Contaminated Soils by Solvent Flushing
}

\author{
By Denie C. M. Augustijn, ${ }^{1}$ Ron E. Jessup, ${ }^{2}$ P. Suresh C. Rao, ${ }^{3}$ \\ and $A$. Lynn Wood ${ }^{4}$
}

\begin{abstract}
Solvent flushing is a potential technique for remediating a waste disposal/spill site contaminated with organic chemicals. This technique involves the injection of a solvent mixture (e.g., water plus alcohols) that enhances contaminant solubility, reduces the retardation factor, and increases the release rates of the contaminants. A simulation model is developed to predict contaminant elution curves during solvent flushing for the case of one-dimensional, steady flow through a contaminated medium. Column experiments are conducted with a Eustis fine sand that is initially equilibrated with an aqueous naphthalene solution, and then eluted with different methanol-water mixtures to remove the naphthalene. The model simulations, based on parameter values estimated from literature data, agree well with the measured elution profiles. Solvent flushing experiments, where the soil was initially equilibrated with a solution of naphthalene and anthracene, show that compounds with different retardation factors are separated at low cosolvent contents, while coelution of the compounds occurs at higher contents. In general, the smaller the retardation factor in water and the higher the cosolvent fraction, the faster the contaminant is recovered. The presence of nonequilibrium conditions, soil heterogeneity, and type of cosolvent will influence the time required to recover the contaminant.
\end{abstract}

\section{INTRODUCTION}

With increasing awareness of the limitations to "pump-and-treat" methods for effective cleanup of ground water contaminated with organic pollutants, interest exists in a new remediation techniques based on chemical additives that increase the efficiency of rate at which contaminants are removed (Palmer and Fish 1992). In situ solvent flushing is one such remediation technique that involves the injection of a mixed solvent (water plus miscible organic cosolvents) at a site contaminated with organic chemicals. The contaminated zone may be located in either the vadoze zone or the saturated zone or both, and can therefore include soils, subsoils, and aquifers. In this paper, the term "soil" will be used in a generic fashion to indicate any of these three media. Site remediation efforts may involve only the highly contaminated "source" area, or may focus on treating the "contaminant plume" emanating from the source. Solvent flushing would be most logically applied for cleanup of the source area, since removal of the source prevents further contamination of ground water. Excavation of the soil from the source area is an alternative option, but the contaminated soil still requires treatment (e.g., incineration, solvent washing) before it can

\footnotetext{
${ }^{1}$ Grad. Res. Asst., Soil and Water Sci. Dept., Univ. of Florida, Gainesville, FL 32611. FL.

${ }^{2}$ Sr. Systems Analyst, Soil and Water Sci. Dept., Univ. of Florida, Gainesville,

${ }^{3}$ Prof., Soil and Water Sci. Dept., Univ. of Florida, Gainesville, FL.

${ }^{4}$ Soil Sci., R. S. Kerr Envir. Res. Lab., U.S. EPA, Ada, OK 74820.

Note. Discussion open until July 1, 1994. To extend the closing date one month, a written request must be filed with the ASCE Manager of Journals. The manuscript for this paper was submitted for review and possible publication on September 8, 1992. This paper is part of the Journal of Environmental Engineering, Vol. 120, No. 1, January/February, 1994. CASCE, ISSN 0733-9372/94/0001-0042/ $\$ 1.00+\$ .15$ per page. Paper No. 4740.
} 
be safely disposed. Excavation can also be very expensive, especially when a large volume of soil is contaminated, and may not even be possible when, for example, the source is located under permanent structures. In such cases, solvent flushing may be considered as a more plausible alternative.

In situ solvent flushing involves the following steps ("In situ" 1991): (1) The solvent mixture is injected upstream of the contaminated zone; (2) the solvent with the dissolved contaminants is extracted downstream and treated above ground to recover the solvent; and (3) the recovered solvent may be reinjected. An impermeable layer as well as physical barriers (e.g., sheet piling, slurry walls) or hydraulic control measures may be required to prevent off-site transport of contaminants and solvents. A schematic representation of this technique is shown in Fig. 1.

The expectation that water-miscible organic cosolvents can be used for enhanced in situ remediation is based on three observations. First, addition of cosolvents leads to an increase in solubility of the nonpolar organic contaminants. Earlier studies established the log-linear enhancement in the solubility of hydrophobic organic chemicals when a cosolvent is added to an aqueous solution (Yalkowsky and Roseman 1981; Fu and Luthy 1986a; Morris et al. 1988). Second, since sorption is inversely related to solubility, sorption will decrease in a proportional log-linear manner upon addition of a cosolvent (Rao et al. 1985; Nkedi-Kizza et al. 1985; Fu and Luthy 1986b). Third, addition of cosolvents has been shown to decrease nonequilibrium sorption constraints (Nkedi-Kizza et al. 1989; Wood et al. 1990; Brusseau et al. 1991b). Cosolvents have been used to facilitate laboratory investigation of sorption and transport of low-solubility organic chemicals in soils (Rao et al. 1991), and for studying the behavior of complex organic wastes such as coal tar (Lane and Loehr 1992). The use of cosolvents for extraction of organic contaminants from various environmental matrices has been successfully demonstrated either at the pilot-scale or in full-scale commercial applications ("Solvent" 1990).

In this paper, a simulation model is presented for predicting solute elution profiles from a contaminated soil that is subjected to solvent flushing. The model assumes a water-saturated soil initially in equilibrium with a contaminant solution that is then flushed with a solvent mixture. Contaminant sorption and transport in the soil are modified as the mixed solvent front

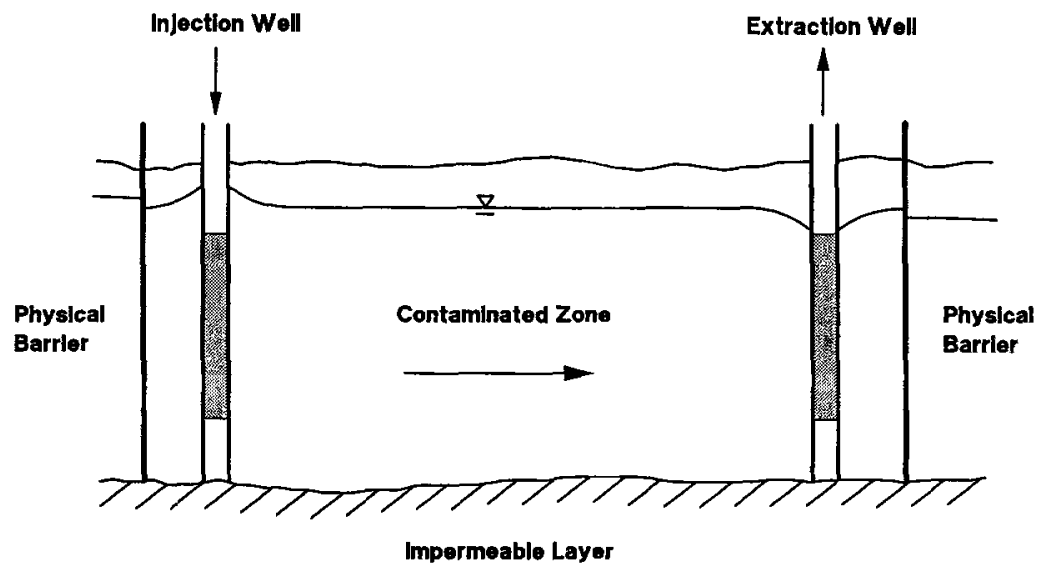

FIG. 1. Schematic Representation of In Situ Solvent Flushing Technique 
advances through the soil, displacing the resident pore water. The model serves as a first approximation in understanding and estimating the effects of cosolvent injection at a waste disposal/spill site. Data from column studies will be presented to validate the model simulations.

\section{THEORY}

\section{Transport}

For a field-scale application of solvent flushing, three-dimensional solute transport in a heterogeneous flow domain should be considered. The focus of the present investigation is to examine cosolvent effects, not the impacts of flow heterogeneities. Therefore, for simplicity, the advective-dispersive transport of the cosolvent (assumed to be nonsorbed) and the contaminant are assumed to occur in a homogeneous medium under steady, one-dimensional flow conditions. Contaminant sorption is described by a linear, reversible isotherm (Chiou et al. 1983). A first-order bicontinuum mass-transfer model [see Selim et al. (1976); Cameron and Klute (1977); Brusseau and Rao (1989a)] was used to describe sorption nonequilibrium during flow.

The dimensionless transport equations used to describe the elution of the cosolvent and the contaminant under specified initial and boundary conditions are summarized in Appendix I. The initial conditions [(6) and (7)] infer equilibrium between the solution and sorbed phase as well as uniform contaminant concentration throughout the column. At $t=0$, a "clean" solvent mixture is introduced at the column inlet. Flux-type boundary conditions [(8) and (9)] are assumed applicable both at the column inlet and outlet (Van Genuchten and Wierenga 1986).

The nondimensional formulation of the bicontinuum model presented here, differs from earlier versions (e.g., Brusseau et al. 1991b) in several ways. The solution-phase and sorbed-phase concentrations $\left(C^{*}\right.$ and $\left.S^{*}\right)$ are scaled to the total contaminant mass initially present in the column [(10) and (11)]. Thus, the area under the elution curve equals 1 when complete recovery of the contaminant is achieved. Since the soil is assumed to be in equilibrium with the contaminant solution, the initial relative concentration in the solution equals the reciprocal value of the retardation factor in water [(6)]. Hence, the relative concentration in the column effluent during the early stages of elution serves as a convenient estimator of the aqueous retardation factor. Also, note that $C^{*}=1$ corresponds to the case where all of the initial sorbed contaminant mass in a volume element of the soil desorbs instantaneously into the solution phase. The Damkohler number $(\omega)$ represents the ratio of advective-flow residence time $(L / v)$ to characteristic sorption time $\left(1 / k_{2}\right)$, and is defined independent of $R$ and $F$ in contrast to the more common definition for $\omega$ (Brusseau and Rao 1991a).

Eqs. (4) and (5) in Appendix I were solved using a Crank-Nicolson central finite difference method (Wang and Anderson 1982) for the given initial and boundary conditions. A numerical approximation for the solution to the advective-dispersive solute transport model presented by Brenner (1962) was used to predict the cosolvent composition in the column. As the mixed solvent front progresses through the soil column, cosolvent effects on sorption are reflected by the changing values of three model parameters: the retardation factor $(R)$; the fraction of instantaneous sorption $(F)$; and the Damkohler number $(\omega)$ for sorption nonequilibrium. The basis for modeling such changes is discussed in the following sections. 


\section{Equilibrium Sorption}

Several studies have shown that the equilibrium sorption coefficient $\left(K_{p}\right)$ decreases in a log-linear manner with increasing cosolvent fraction (Rao et al. 1985; Nkedi-Kizza et al. 1985; Fu and Luthy 1986b). Hence, the change in retardation factor $(R)$ as a function of cosolvent fraction can be described by (Nkedi-Kizza et al. 1987):

$\log \left(R_{m}-1\right)=\log \left(R_{w}-1\right)-\alpha \beta \sigma f_{c}$

where the subscripts $m$ and $w=$ mixed solvent and water, respectively; $\sigma$ $=$ the cosolvency power, defined by Pinal et al. (1990) as $\log \left(S_{c} / S_{w}\right)$, where $S_{c}$ and $S_{w}=$ solubilities in the pure (neat) organic solvent and water, respectively; and $f_{c}=$ the volume fraction of organic cosolvent. The empirical coefficients $\alpha$ and $\beta$ account for deviations of the measured cosolvency power from the theoretical value as a result of solvent-sorbent and solvent-cosolvent interactions, respectively. Such interactions may either enhance $(\alpha \beta$ $>1$ ) or diminish $(\alpha \beta<1)$ the effect of cosolvents on sorption (Rao et al. 1991).

\section{Sorption Nonequilibrium}

The bicontinuum model has been used successfully to describe diffusionlimited sorption of hydrophobic organic chemicals during transport in soils (Nkedi-Kizza et al. 1989; Brusseau and Rao 1989a; Brusseau at al. 1990; 1991a, 1991b). In this model, a fraction of the sorption is assumed to occur in an instantaneous manner, while the remainder is described by a firstorder mass transfer model. Rate-limited sorption has been attributed to solute diffusion constraints within the interior regions of organic matter (Brusseau et al. 1991a) or to retarded intraparticle diffusion (Wu and Gschwend 1986; Ball and Roberts 1991).

Based on an analysis of literature data, Brusseau and Rao (1989b) presented a log-log, inverse linear relationship between the equilibrium sorption coefficient $\left(K_{p}\right)$ and the reverse first-order sorption rate coefficient $\left(k_{2}\right)$. Such trends were confirmed in later investigations (Brusseau et al. 1990, 1991a). The reciprocal linear relationship between $\log k_{2}$ and $\log K_{p}$, and the linear decrease in $\log K_{p}$ with increasing cosolvent content $\left(f_{c}\right)$ suggest that $k_{2}$ would increase in a log-linear fashion as the cosolvent content increases. Brusseau et al. (1991b) presented data confirming this relationship. Based on these findings, the increase in the value for the Damkohler number $(\omega)$ with cosolvent content may be calculated using the following equation:

$\log \omega_{m}=\log \omega_{w}+a \alpha \beta \sigma f_{c}$

where $a=$ the value for the slope of the $\log k_{2}$ versus $\log K_{p}$ relationship and other parameters are as defined before. Note that $a=0.668$ based on the analysis of Brusseau and Rao (1989b).

Brusseau et al. (1991b) also noted changes in the parameter $F$ as the cosolvent content was increased. The general trend was that up to a certain volume percent cosolvent $(\sim 20 \%)$ no significant change was found in $F$, while at higher cosolvent fractions a decrease in $F$ was observed. Here, we will assume an initial constant $F$ and a linear decrease beyond a cosolvent fraction of 0.2 to an arbitrary value of 0.01 for the neat organic solvent

$$
\begin{aligned}
& F_{m}=F_{w} \quad 0 \leq f_{c}<0.2 \quad \ldots \ldots \ldots \ldots \\
& F_{m}=F_{w}-b\left(f_{c}-0.2\right) \quad 0.2 \leq f_{c}<1
\end{aligned}
$$


where $b=\left(F_{w}-0.01\right) / 0.8$. Since $F$ changes only over a relative small range the model is insensitive to the function of $F$ for $f_{c}$.

\section{Dynamics of Solvent Flushing}

In Fig. 2, simulated curves for naphthalene elution by two methanolwater mixtures are shown for equilibrium and nonequilibrium conditions. Also shown is the normalized breakthrough curve for methanol, which is assumed to behave as a nonreactive tracer. The following general features of the contaminant elution curves are evident: (1) For the initial portion of the curve (up to one pore volume effluent) the naphthalene concentration is constant; this corresponds to a displacement of the resident aqueous solution by the incoming mixed solvent; the magnitude of the relative effluent concentration during this period is equal to $1 / R_{w}$, as defined in (6); (2) the area under the contaminant breakthrough curves at all cosolvent contents is the same, and equals unity; (3) coincident with the breakthrough of the cosolvent, the contaminant concentration rises sharply, and then declines gradually to zero; (4) with increasing cosolvent fraction, the peak concentration is greater and the breakthrough curve is increasingly symmetrical and more compressed; and (5) at higher cosolvent fractions (50\% in this example) the relative effluent concentration $\left(C^{*}\right)$ exceeds 1 , indicating that mass is compressed into a small liquid volume.

Increasing peak height and narrowing band width is a well-known effect of gradient elution in liquid chromatography (Snyder and Kirkland 1979). A similar effect was reported in the cation exchange literature, and is referred to as the "snowplow" effect (Starr and Parlange 1979). This effect can be attributed to a change in the sorption coefficient as the solvent composition changes during an elution experiment. The mixed solvent front passing through the column favors desorption so strongly that most of the sorbed mass desorbs immediately into solution and is transported along with the mixed solvent causing an accumulation of solute at the solvent front. The compression of contaminant mass in a small liquid volume is very convenient for remediation purposes since it reduces the number of pore

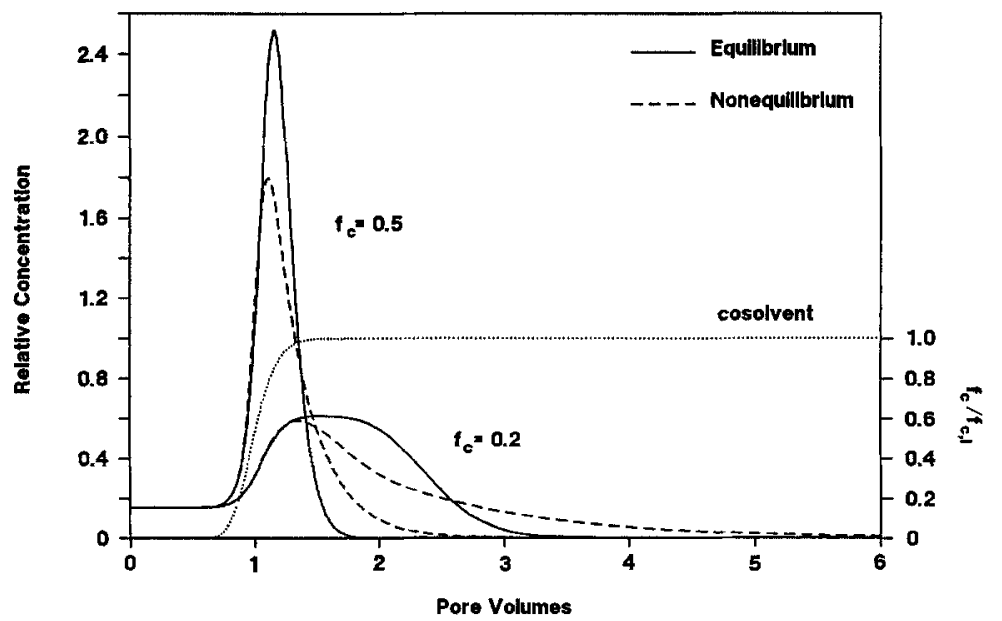

FIG. 2. Simulated Elution Profiles of Naphthalene for $\mathbf{2 0} \%$ and $\mathbf{5 0} \%$ Methanol Fractions 
volumes required to remove the contaminant from the soil. Fig. 2, for example, shows that the naphthalene can be removed with only one injected pore volume of a $50 \%$ methanol fraction, assuming equilibrium conditions.

To further illustrate the effects of contaminant elution from soils, Fig. 3 shows simulated concentration profiles of naphthalene within the column for the solution [Fig. 3(a)] and sorbed phase [Fig. 3(b)] at two different times and a methanol fraction of 0.2 . The nonequilibrium curves shown in Figs. 2 and 3 are very similar to those for equilibrium conditions for the initial portion of the elution profiles, while the peak concentration is lower and the distal portion of the elution curve exhibits more tailing for nonequilibrium conditions. As the high concentrations move forward in the column, sorption occurs along the mixed solvent front [sorbed concentrations at the solvent front are higher than the initial concentrations in the sorbed phase, see Fig. 3(b)]. Under equilibrium conditions sorption is instantaneous; however, under nonequilibrium conditions sorption occurs slower causing the contaminant to travel faster. For this reason, contaminant concentrations at the front of the elution profiles under nonequilibrium con-

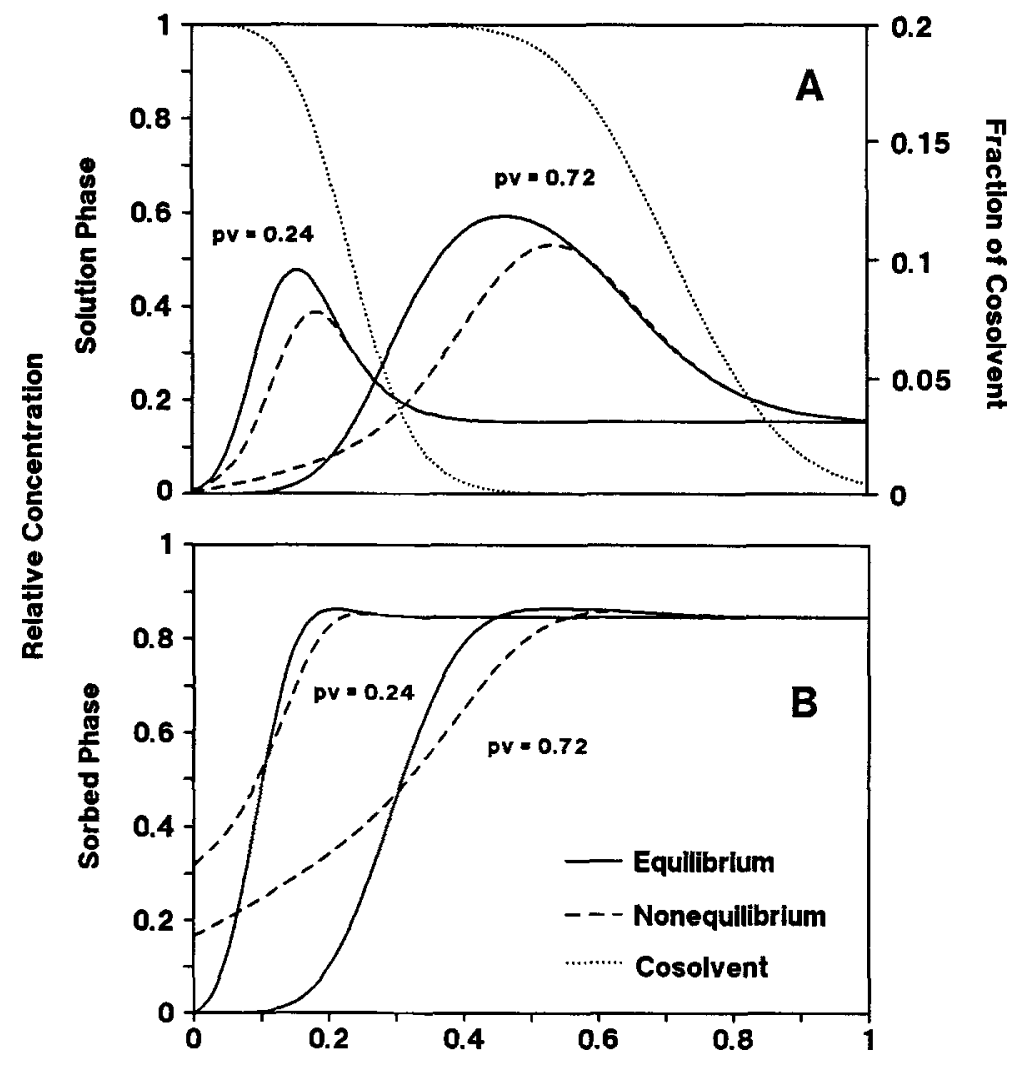

Relative Distance in the Column

FIG. 3. Simulated Concentration Profiles within Column for Naphthalene at Two Different Pore Volumes ( $p v$ ) in Solution (a) Sorbed Phase; (b) at Methanol Fraction of $\mathbf{2 0} \%$ 
ditions are similar to, or even higher than, those for equilibrium conditions. As the front passes, the cosolvent fraction increases favoring desorption. Since desorption is rate-limited under nonequilibrium conditions, this results in tailing of the distal end of the elution curves. It is evident that nonequilibrium conditions reduce the efficiency of contaminant elution.

In Figs. 2 and 3(a), the profiles of the cosolvent are also included. Recall that the values for $R, F$, and $\omega$ will change along the dispersed portion of the solvent front. In heterogeneous soils, this solvent gradient portion extends over a larger number of pore volumes (or distance), and will result in broader contaminant elution profiles. Thus, solvent flushing is expected to be less efficient in heterogeneous field soils.

\section{MATERIALS AND METHODS}

The experimental procedure used in this study is modified from the miscible displacement technique described by Brusseau et al. (1990). An airdry sample of Eustis fine sand was packed in a glass column (diameter 2.5 $\mathrm{cm}$, length $5.3 \mathrm{~cm}$ ). This was the same soil as used by Brusseau et al. (1991b) and had an organic carbon content of $0.39 \%$ by weight. The packed soil had a dry bulk density of $1.75 \mathrm{~g} / \mathrm{cm}^{3}$ and a porosity of $0.33 \mathrm{~cm}^{3} / \mathrm{cm}^{3}$. The column was saturated overnight by flushing with an aqueous $0.01 \mathrm{~N} \mathrm{CaCl}_{2}$ solution. Pentafluorobenzoic acid (PFBA) was used as a nonreactive tracer to determine the hydrodynamic dispersion characteristics of the water-saturated column. The breakthrough curve for a pulse of PFBA was used to estimate the Peclet number for the soil column $(P=84)$.

The soil column was then saturated with an aqueous napthalene solution (about $15 \mathrm{mg} / \mathrm{L}$ ) in a $0.01 \mathrm{~N} \mathrm{CaCl}_{2}$ matrix. Naphthalene concentration in the column effluent was tracked using a flow-through ultraviolet (UV) detector (Gilson Holochrome). The detector response was recorded on a strip chart recorder (OmniScribe D5000). The naphthalene solution was applied until the signal from the detector reached a plateau equal to the signal of the input concentration. The flow was then interrupted for approximately 12 hours. When the flow was resumed a slight decrease was observed in the response of the detector, indicating that the concentration had decreased during the quiescent period. This indicates that equilibrium was not established before the flow was interrupted (Brusseau et al. 1989). The naphthalene solution was applied for at least another 3 hours at a flow rate of $1 \mathrm{~mL} / \mathrm{min}(v=37 \mathrm{~cm} / \mathrm{hr})$. After this, it was assumed that equilibrium was established in the column, satisfying the initial condition assumed for the model.

The solvent flushing experiment was initiated by introducing a mixed solvent (methanol plus water) at the column inlet. The column effluent was collected in 4-mL amber vials for HPLC analysis for naphthalene (Acetonitrile/water $50 / 50$ mobile phase; liquid chromatography polyaromatic hydrocarbons (LCPAH) column). At times when the naphthalene concentration in the effluent was expected to change rapidly, smaller volume fractions were collected in neat methanol to avoid volatilization losses of naphthalene; otherwise, all effluent samples were collected until the vials were filled and no headspace remained. All vials were immediately capped with screw caps fitted with Teflon septa. Naphthalene elution experiments were done with $0.01 \mathrm{~N} \mathrm{CaCl}_{2}$ solutions containing 10,20 , and $30 \%$ (by volume) methanol at a pore water velocity of about $70 \mathrm{~cm} / \mathrm{hr}$. Between each experiment the soil was reequilibrated with an aqueous naphthalene solution for at least 12 hours. 
The same procedure was followed to conduct additional experiments where the soil was initially equilibrated with an aqueous solution of naphthalene and anthracene prior to elution with $30 \%$ and $70 \%$ methanol solutions.

\section{RESULTS}

The experimental data for elution of naphthalene at three cosolvent fractions are shown in Fig. 4. Independent predictions were done for each curve using the solvent flushing model. The column parameters were obtained experimentally, values for the cosolvency powers were taken from Pinal et al. (1990), and all other transport and cosolvency parameters were obtained from regression analyses using the data of Brusseau et al. (1991b). A summary of the parameter values is given in Tables 1 and 2. The Peclet number was assumed to be constant for all cosolvent fractions. This assumption was based on experiments by Wood et al. (1990), who showed that cosolvents had very little effect on the hydrodynamic parameters for the packed material. The experimental data show all characteristic features of typical elution curves as discussed before. Considering the independent estimation of parameter values, the simulations agree reasonably well with the experimental data, except that at $20 \%$ and $30 \%$ methanol fractions the model overpredicts the peak concentrations of the elution profiles. The model is most sensitive to the value for the retardation factor in the solvent mixture $\left(R_{m}\right)$. A small reduction in $R_{m}$ results in lower peak concentrations and more dispersed elution curves. Given the large confidence intervals associated with $R_{w}$ and $\alpha \beta$ (Table 1), which determine $R_{m}$ [(1)], the fit to the experimental data may be considered reasonable.

When solvent flushing is applied to sites contaminated with multiple components, the compounds may become separated at lower cosolvent fractions as a result of large differences between retardation factors and sorption nonequilibrium parameters (see Table 1). This behavior is illustrated in Fig. $5(a)$, where experimental data and predicted elution curves are shown for naphthalene and anthracene at a cosolvent fraction of $30 \%$. At higher cosolvent fractions, however, such chromatographic separation is negligibly small as shown in Fig. 5(b). At 70\% methanol, retardation factors for both naphthalene and anthracene are reduced to values close to 1 , causing the compounds to coelute at an earlier time. This behavior is well known in liquid chromatography where the mobile phase composition is chosen to optimize separation of compounds and minimize elution times (Snyder and Kirkland 1979). The experimental data agree reasonably well with the simulated trends. It is therefore evident that higher cosolvent fractions are most efficient for remediation of a contaminated site. The data collected for naphthalene elution at $30 \%$ methanol [Fig. 4(c)] are also included in Fig. $5(a)$ as open circles. The agreement between the data collected from two separate experiments indicates a good reproducibility of the experimental data.

\section{ANALYSIS}

The soil (Eustis fine sand), organic solutes (naphthalene and anthracene), and the cosolvent (methanol) used in this study were chosen primarily because the required data for sorption from mixed solvents were available from the literature (Brusseau et al. 1991b). The following factors should be considered in extrapolating our results to other systems. 

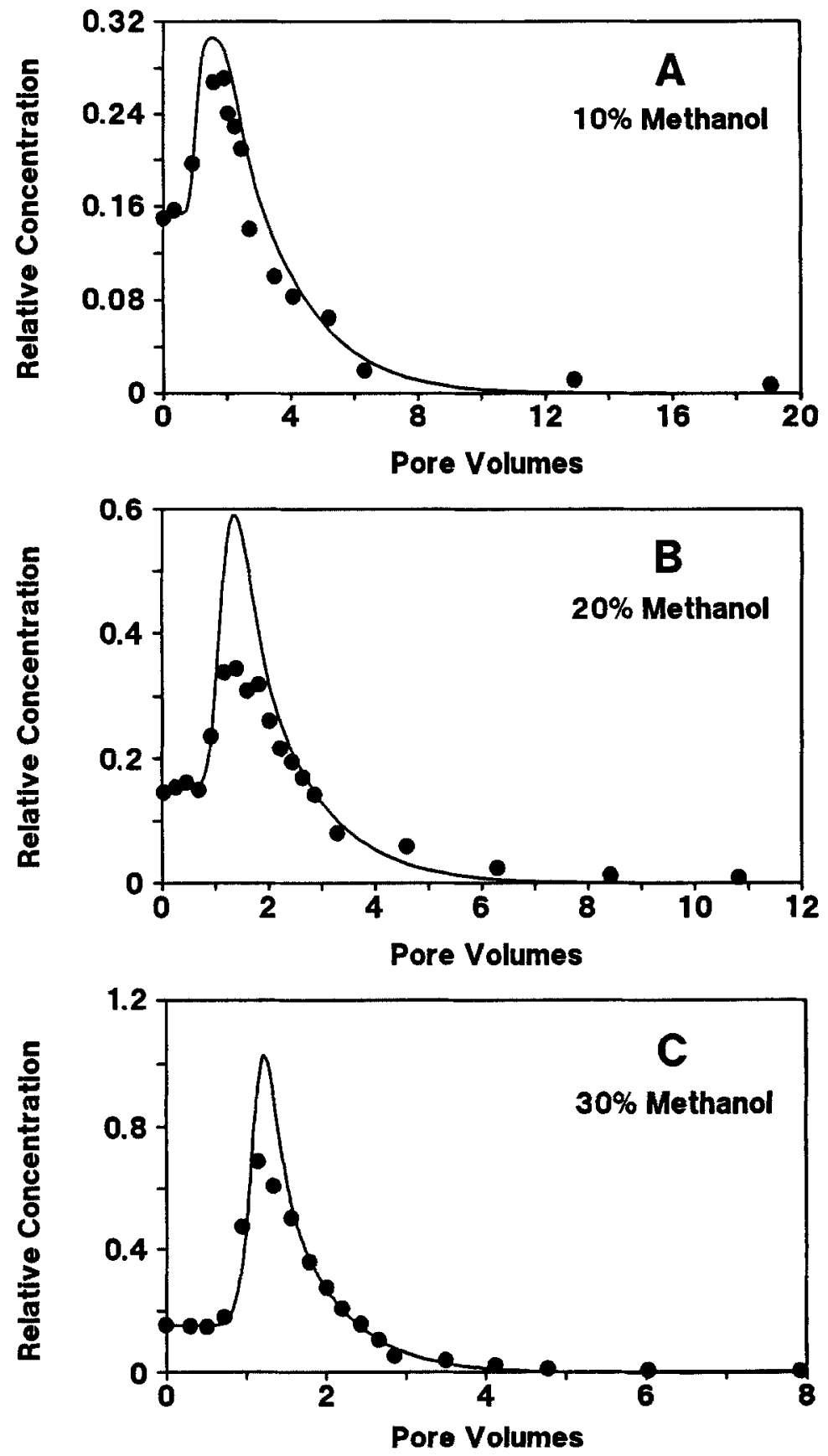

FIG. 4. Experimental Data (Filled Circles) and Independent Model Predictions (Solid Lines) for Naphthalene Elution Curves at Three Different Cosolvent Fractions 
TABLE 1. Column Parameter Values for Elution of Naphthalene and Anthracene with Methanol-Water Mixtures from Eustis Fine Sand

\begin{tabular}{l|c|c}
\hline \hline $\begin{array}{c}\text { Parameter } \\
(1)\end{array}$ & $\begin{array}{c}\text { Value } \\
(2)\end{array}$ & $\begin{array}{c}95 \% \text { Confidence Interval } \\
(3)\end{array}$ \\
\hline$v(\mathrm{~cm} / \mathrm{hr})$ & 70 & - \\
$L(\mathrm{~cm})$ & 5.3 & - \\
$\rho\left(\mathrm{g} / \mathrm{cm}^{3}\right)$ & 1.75 & - \\
$\theta\left(\mathrm{cm}^{3} / \mathrm{cm}^{3}\right)$ & 0.33 & $78-93$ \\
$P$ & 84 & \\
\hline
\end{tabular}

TABLE 2. Transport and Cosolvency Parameter Values for Elution of Naphthalene and Anthracene with Methanol-Water Mixtures from Eustis Fine Sand

\begin{tabular}{c|c|c|c|c}
\hline \multirow{2}{*}{\begin{tabular}{c} 
Parameter \\
\cline { 2 - 5 }$(1)$
\end{tabular}} & $\begin{array}{c}\text { Value } \\
(2)\end{array}$ & $\begin{array}{c}95 \% \text { confidence } \\
\text { interval } \\
(3)\end{array}$ & $\begin{array}{c}\text { Aalue } \\
(4)\end{array}$ & $\begin{array}{c}95 \% \text { confidence } \\
\text { interval } \\
(5)\end{array}$ \\
\hline$R_{w}$ & 6.5 & $3.2-14.6$ & 229 & $58-922$ \\
$\omega_{w}$ & 0.57 & $0.50-0.65$ & 0.03 & $0.005-0.2$ \\
$F_{w}$ & 0.46 & $0.42-0.50$ & 0.52 & $0.34-0.70$ \\
$\sigma$ & 3.42 & - & 4.06 & - \\
$\alpha \beta$ & 0.87 & $0.26-1.48$ & 1.00 & $0.67-1.33$ \\
$a$ & 0.59 & $0.07-1.11$ & 0.87 & $0.71-1.03$ \\
\hline \hline
\end{tabular}

Hydrologic considerations suggest that the in situ solvent flushing technique is likely to be more successful for contaminated sites with coarsetextured soils (or aquifer solids). Low hydraulic conductivities for the finertextured materials would limit the solvent flushing rates, and thus require longer times for complete contaminant elution.

Solvent flushing would be more appropriate for more hydrophobic chemicals, such as polychlorinated biphenyls and larger polynuclear aromatic hydrocarbons, for which most other remediation techniques are inefficient. Sites contaminated with several compounds or complex waste mixtures may also be considered for solvent flushing, since the coelution of small and larger compounds is favored at higher cosolvent contents [Fig. 5(b)].

In this study, experiments were performed with methanol as the cosolvent. Other water-miscible cosolvents (e.g., ethanol or acetone) are expected to give similar results. The efficiency of each cosolvent is determined by the value of $\alpha \beta \sigma$; the larger this value, the higher is the recovery efficiency for given cosolvent fraction. In general, partially miscible organic solvents, such as butanone, are much stronger solvents (i.e., larger $\sigma$ ) than completely miscible organic solvents like methanol (Pinal et al. 1990). A disadvantage of partially miscible organic solvents, however, is the limited solubility in water: therefore, a mixture of solvents may be most efficient for soil remediation by in situ solvent flushing.

When injecting a cosolvent at a site, caution needs to be taken to avoid leaching of the cosolvent outside the hydraulically controlled zone that may facilitate offsite transport of the contaminants. Also, most cosolvents themselves are hazardous chemicals, and should be prevented from leaving the hydraulically controlled zone. After the target contaminants have been recovered, the mixed solvent can be displaced from the soil by flushing with 

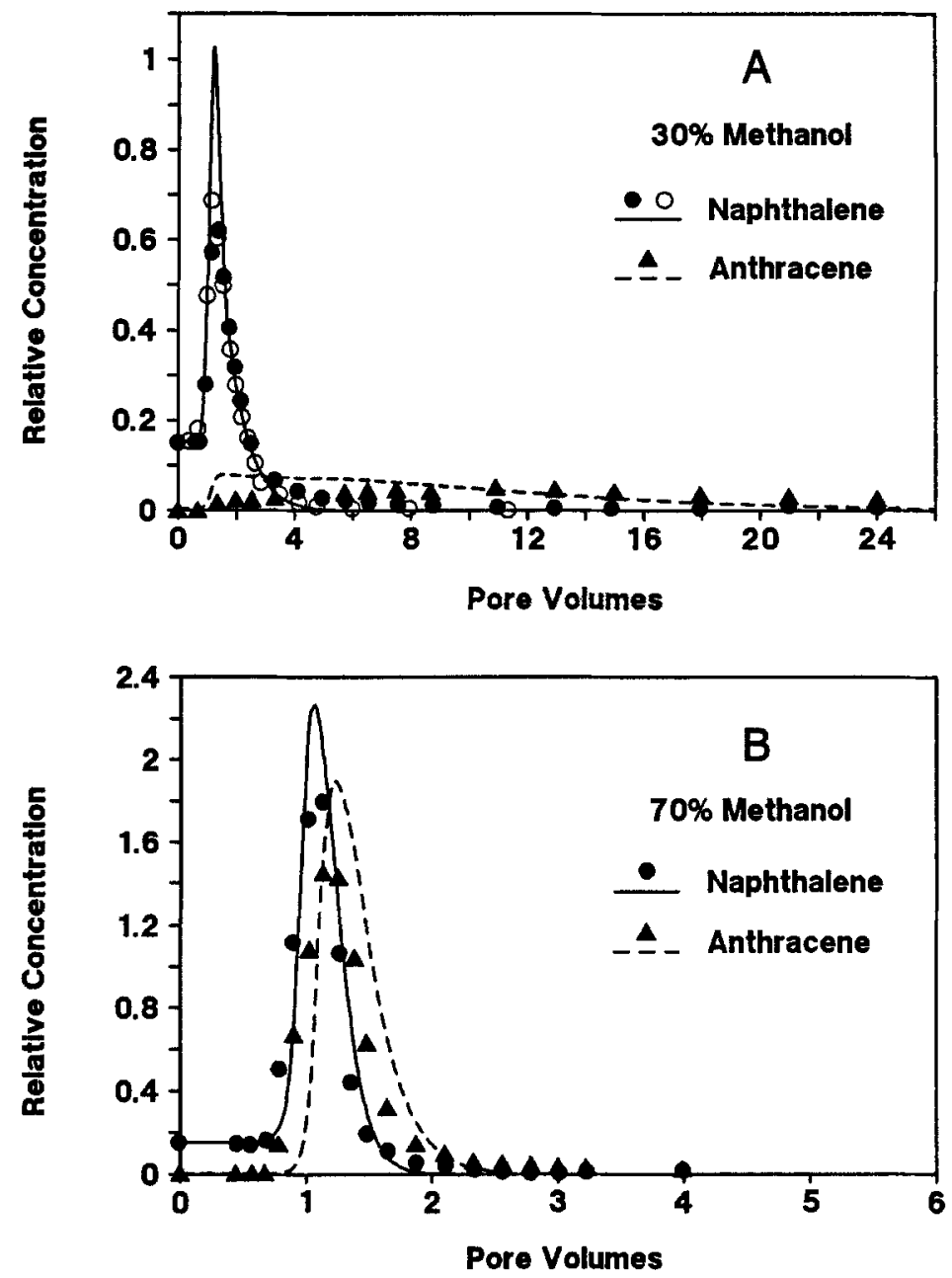

FIG. 5. Experimental Data (Symbols) and Independent Model Predictions (Lines) for Binary Solute Elution Experiments at Two Different Cosolvent Fractions

water. Since the cosolvent is not retained by the soil, this should not take more than a few pore volumes. Any residual amounts of cosolvent would likely be biodegraded by native microorganisms, assuming recovery and reestablishment of appropriate microbial consortia. In addition to the efficiency and toxicity of the cosolvent, the costs of the operation should be considered in selecting the best cosolvent or combination of cosolvents.

The elution experiments were done at high velocities to induce nonequilibrium conditions. In a realistic field experiment, much slower velocities will likely be employed. Simulations performed with velocities 10 times smaller than those used in the experiments showed that near-equilibrium conditions would be approached [large $\omega ;(16)]$. An advantage of this is that model parameters pertaining to sorption nonequilibrium ( $\omega$ and $F$ ) need not be determined. Equilibrium conditions are even more valid at higher 
cosolvent fractions. A recent study by Brusseau (1992), however, showed that $k_{2}$ values decreased when slower velocities were used. Although this behavior should be studied further and better quantified, it may affect extrapolation of data from high velocity experiments to the lower flow velocities expected in field studies. In addition, for contaminated soils in the field it is also possible that direct contact of pure products or aging may affect the desorption kinetics of the organic solutes. Also, physical nonequilibrium conditions due to soil heterogeneity may be more important under field conditions, as discussed before.

This study shows that solvent flushing is an effective technique to remove organic solutes from contaminated soils. The model presented here provided reasonable predictions of the contaminant elution curves from artificially contaminated soils. This model may be used in designing a solvent-flushing remediation scheme and optimizing its efficiency. One has to recognize, however, the limitations of assumptions made in the model, when interpreting the model outputs. Also, the accuracy of the model predictions depends greatly on the uncertainty in input parameters. More complete models (e.g., three-dimensional flow, including soil heterogeneity and unsaturated flow conditions) are necessary to evaluate the full range of problems associated with field-scale applications of solvent flushing.

\section{SUMMARY AND CONCLUSIONS}

A one-dimensional contaminant transport model describing the elution of organic pollutants from contaminated soils using mixed solvents was presented. The model makes use of the cosolvency theory which has been well established over the past several years. The decrease in retardation factor $(R)$, and increase in sorption rate coefficient $\left(k_{2}\right)$, with increasing volume fraction of cosolvent $\rightarrow\left(f_{c}\right)$ were described by a log-linear relationship. An empirical two-step linear function was chosen for the relationship between the fraction of instantaneous sorption domains $(F)$ and $f_{c}$.

Laboratory miscible displacement experiments were conducted to validate the model predictions. A column packed with Eustis fine sand was equilibrated with an aqueous naphthalene solution and flushed with three methanol-water mixtures of different compositions. Two displacement experiments were conducted with naphthalene and anthracene as representative nonpolar organic contaminants to investigate the effects of solvent flushing on soils contaminated with multiple compounds. Independent predictions with the model showed good agreement with the experimental data, which supports the applicability of the model.

Experimental data and model simulations showed that with increasing cosolvent content the contaminant eluted at higher concentrations, thus improving the contaminant recovery efficiency. The experiments conducted with two solutes showed separation of the compounds at low cosolvent fractions, while at higher fractions coelution occurred at relative early times as a result of strongly reduced retardation factors. This indicates that high cosolvent fractions are most efficient in the elution of contaminants. The recovery efficiency is expected to reduce in the presence of nonequilibrium conditions and soil heterogeneity. The composition of the solvent mixture and type of cosolvent can be used as design parameters to optimize the recovery efficiency.

The presented model is useful in understanding the effects of cosolvents when used for remediation of contaminated soils, and has potential application for designing in situ solvent flushing techniques. 


\section{ACKNOWLEDGMENTS}

This research was partially funded by U.S. Environmental Protection Agency (CR-820678) and Electric Power Research Institute (RP-2879-7). Although the research described in this paper was supported by the U.S. EPA through a cooperative agreement, it has not been subjected to the agency's required peer and policy review, and therefore does not necessarily reflect the views of the agency, and no official endorsement should be inferred. Approved for publication as Florida Agricultural Experiment Station Journal Series No. R-02653.

\section{APPENDIX I. GOVERNING TRANSPORT EQUATIONS FOR SOLVENT FLUSHING MODEL}

General one-dimensional transport equations including a first-order bicontinuum mass-transfer model for sorption:

$$
\begin{aligned}
& \frac{\partial C^{*}}{\partial p}+\frac{\partial F(R-1) C^{*}}{\partial p}+\frac{\partial S^{*}}{\partial p}=\frac{1}{P} \frac{\partial^{2} C^{*}}{\partial X^{2}}+\frac{\partial C^{*}}{\partial X} \\
& \frac{\partial S^{*}}{\partial p}=\omega\left[(1-F)(R-1) C^{*}-S^{*}\right] \ldots \ldots \ldots
\end{aligned}
$$

Initial conditions:

$$
\begin{aligned}
& C^{*}=\frac{1}{R} \quad p=0 ; 0 \leq X \leq 1 \quad \ldots \ldots \ldots \ldots \\
& S^{*}=\frac{(1-F)(R-1)}{R} \quad p=0 ; 0 \leq X \leq 1
\end{aligned}
$$

Boundary conditions:

$$
\begin{aligned}
& C^{*}=\frac{1}{P} \frac{\partial C^{*}}{\partial X}=0 \quad p>0 ; X=0 \\
& \frac{\partial C^{*}}{\partial X}=0 \quad p>0 ; X=1 \ldots \ldots
\end{aligned}
$$

Nondimensional parameters:

$$
\begin{aligned}
& C^{*}=\frac{\theta C}{M} \\
& S^{*}=\frac{\rho S_{2}}{M} \\
& p=\frac{v t}{L} \\
& X=\frac{x}{L} \\
& P=\frac{v L}{D} .
\end{aligned}
$$


$R=1+\frac{\rho}{\theta} K_{p}$

$\omega=\frac{k_{2} L}{v}$

where $C=$ concentration in solution phase $\left(M / L^{3}\right) ; C^{*}=$ relative concentration; $D=$ hydrodynamic dispersion coefficient $\left(L^{2} / T\right) ; F=$ fraction of instantaneous sorption domain; $K_{p}=$ equilibrium sorption coefficient $\left(L^{3 /}\right.$ $M) ; k_{2}=$ reverse first-order sorption rate coefficient $\left(T^{-1}\right) ; L=$ column length $(L) ; M=$ total initial contaminant mass in the column $\left(M / L^{3}\right)=$ $\theta R C_{0}$, where $C_{0}=$ initial concentration $\left(M / L^{3}\right) ; P=$ Peclet number; $p=$ dimensionless time in pore volumes; $R=$ retardation factor; $S_{2}=$ sorbed concentration in the rate-limited domain $(M / M) ; S^{*}=$ normalized sorbed concentration in the rate-limited domain; $t=$ time $(T) ; v=$ pore water velocity $(L / T) ; X=$ relative distance in the column; $x=$ distance $(L) ; \theta$ $=$ volumetric water content $\left(L^{3} / L^{3}\right) ; p=$ dry bulk density $\left(M / L^{3}\right)$; and $\omega$ $=$ Damkohler number.

\section{APPENDIX II. REFERENCES}

Ball, W. P., and Roberts, P. V. (1991). "Long-term sorption of halogenated organic chemicals by aquifer material. 2 . Intraparticle diffusion." Environ. Sci. Technol., 25(7), 1237-1249.

Brenner, H. (1962). "The diffusion model of longitudinal mixing in beds of finite length. Numerical values." Chem. Eng. Sci., 17(4), 229-243.

Brusseau, M. L. (1992). "Nonequilibrium transport of organic chemicals: The impact of pore-water velocity." J. Contam. Hydrol., 9(4), 353-368.

Brusseau, M. L., Jessup, R. E., and Rao, P. S. C. (1990). "Sorption kinetics of organic chemicals: Evaluation of gas-purge and miscible-displacement techniques." Environ. Sci. Technol., 24(5), 727-735.

Brusseau, M. L., Jessup, R. E., and Rao, P. S. C. (1991a). "Nonequilibrium sorption of organic chemicals: Elucidation of rate-limiting processes." Environ. Sci. Technol., 25(1), 134-142.

Brusseau, M. L., and Rao, P. S. C. (1989a). "Sorption nonideality during organic contaminant transport in porous media." CRC Crit. Rev. Environ. Control, 19(1), $33-99$.

Brusseau, M. L., and Rao, P. S. C. (1989b). "The influence of sorbate-organic matter interactions on sorption nonequilibrium." Chemosphere, 18(9/10), 16911706 .

Brusseau, M. L., Rao, P. S. C., Jessup, R. E., and Davidson, J. M. (1989). "Flow interruption: A method for investigating sorption nonequilibrium." J. Contam. Hydrol., 4(3), 223-240.

Brusseau, M. L., Wood, A. L., and Rao, P. S. C. (1991b). "Influence of organic cosolvents on the sorption kinetics of hydrophobic organic chemicals." Environ. Sci. Technol., 25(5), 903-910.

Cameron, D. R., and Klute, A. (1977). "Convective-dispersive solute transport with a combined equilibrium and kinetic adsorption model." Water Resour. Res., 13(1), $183-188$.

Chiou, C. T., Porter, P. E., and Schmedding, D. W. (1983). "Partition equilibria of nonionic organic compounds between soil organic matter and water." Environ. Sci. Technol., 17(4), 227-231.

Fu, J., and Luthy, R. G. (1986a). "Aromatic compound solubility in solvent/water mixtures." J. Envir. Engrg., ASCE, 112(2), 328-345.

$\mathrm{Fu}$, J., and Luthy, R. G. (1986b). "Effect of organic solvent on sorption of aromatic solutes onto soils." J. Envir. Engrg., ASCE, 112(2), 346-366.

"In situ soil flushing." (1991). Engrg. Bulletin, EPA/540/2-91/021, Office of Emergency and Remedial Response, U.S. Envir. Protection Agency, Washington, D.C. 
Lane, W. F., and Loehr, R. C. (1992). "Estimating the equilibrium aqueous concentrations of polynuclear aromatic hydrocarbons in complex mixtures." Environ. Sci. Technol., 26(5), 983-990.

Morris, K. R., Abramowitz, R., Pinal, R., Davis, P., and Yalkowsky, S. H. (1988). "Solubility of aromatic pollutants in mixed solvents." Chemosphere, 17(2), 285298.

Nkedi-Kizza, P., Brusseau, M. L., Rao, P. S. C., and Hornsby, A. G. (1989). "Nonequilibrium sorption during displacement of hydrophobic organic chemicals and ${ }^{45} \mathrm{Ca}$ through soil columns with aqueous and mixed solvents." Environ. Sci. Technol., 23(7), 814-820.

Nkedi-Kizza, P., Rao, P. S. C., and Hornsby, A. G. (1985). "Influence of organic cosolvents on sorption of hydrophobic organic chemicals by soils." Environ. Sci. Technol., 19(10), 975-979.

Nkedi-Kizza, P., Rao, P. S. C., and Hornsby, A. G. (1987). "Influence of organic cosolvents on leaching of hydrophobic organic chemicals through soils." Environ. Sci. Technol., 21(10), 1107-1111.

Palmer, C. D., and Fish, W. (1992). "Chemical enhancements to pump-and-treat remediation." Ground Water Issue, EPA/540/S-92/001, R. S. Kerr Envir. Res. Lab., U.S. Environ. Protection Agency, Ada, Okla.

Pinal, R., Rao, P. S. C., Lee, L. S., Cline, P. V., and Yalkowsky, S. H. (1990). "Cosolvency and solubility of hydrophobic organic chemicals in mixed solvents: Evaluation using partially- and completely-miscible organic solvents." Environ. Sci. Technol., 24(5), 639-647.

Rao, P. S. C., Hornsby, A. G., Kilcrease, D. P., and Nkedi-Kizza, P. J. (1985). "Sorption and transport of hydrophobic organic chemicals in aqueous and mixed solvent systems: Model development and preliminary evaluation." J. Environ. Qual., 14(3), 376-383.

Rao, P. S. C., Lee, L. S., and Wood, A. L. (1991). "Solubility, sorption, and transport of hydrophobic organic chemicals in complex mixtures." Environ. Res. Brief, EPA-600/M-91/009, U.S. Envir. Protection Agency, Ada, Okla.

Selim, H. M., Davidson, J. M., and Mansell, R. S. (1976). "Evaluation of a twosite adsorption desorption model for describing solute transport in soils." Proc. Summer Computer Simulation Conf., Washington, D.C.

Snyder, L. R., and Kirkland, J. J. (1979). Introduction to modern liquid chromatography, 2d Ed., John Wiley and Sons Inc., New York, N.Y.

"Solvent extraction treatment." (1990). Engrg. Bulletin, EPA/540/2-90/013, Office of Emergency and Remedial Response, U.S. Envir. Protection Agency, Washington, D.C.

Starr, J. L., and Parlange, J.-Y. (1979). "Dispersion in soil columns: The snow plow effect." Soil Sci. Soc. Am. J., 43(3), 448-450.

Van Genuchten, M.Th., and Wierenga, P. J. (1986). "Solute dispersion coefficients and retardation factors." A. Klute, ed., Methods of soil analysis, part 1. physical and mineralogical methods. Agronomy monographs No. 9, 2d Ed., Amer. Society for Agronomy Soil Sci. Soc. of Amer., Madison, Wisc., 1025-1054.

Wang, H. F., and Anderson, M. P. (1982). Introduction to groundwater modeling; finite difference and finite elements methods. Freeman, New York, N.Y.

Wood, A. L., Bouchard, D. C., Brusseau, M. L., and Rao, P. S. C. (1990). "Cosolvent effects on sorption and mobility of organic contaminants in soils." Chemosphere, 21(4/5), 575-587.

Wu, S., and Gschwend, P. M. (1986). "Sorption kinetics of hydrophobic organic compounds to natural sediments and soils." Environ. Sci. Technol., 20(7), 717725 .

Yalkowsky, S. H., and Roseman, T. (1981). "Solubilization of drugs by cosolvents." Yalkowsky, S. H., ed., Techniques of solubilization of drugs, Marcel Dekker Inc., New York, N.Y., 91-134. 


\section{APPENDIX III. NOTATION}

The following symbols are used in this paper:

$a=$ slope of $\log k_{2}$ versus $\log K_{p}$ relationship;

$b=$ empirical constant;

$C=$ concentration in solution $\left(M / L^{3}\right)$;

$C_{0}=$ initial concentration $\left(M / L^{3}\right)$;

$C^{*}=$ normalized concentration;

$D=$ hydrodynamic dispersion coefficient $\left(L^{2} / T\right)$;

$F=$ fraction of instantaneous sorption domains;

$f_{c}=$ volume fraction of cosolvent;

$f_{c, i}=$ volume fraction of cosolvent in the influent;

$K_{p}=$ equilibrium sorption coefficient $\left(L^{3} / M\right)$;

$k_{2}=$ reverse first-order sorption rate coefficient $\left(T^{-1}\right)$;

$L=$ column length $(L)$;

$M=$ total contaminant mass in column $\left(M / L^{3}\right)$;

$P=$ Peclet number;

$p=$ dimensionless time in pore volumes;

$R=$ retardation factor;

$S_{2}=$ sorbed concentration in the rate-limited domain $(M / M)$;

$S^{*}=$ normalized sorbed concentration;

$t=$ time $(T)$

$v=$ pore water velocity $(L / T)$

$X=$ relative distance;

$x=$ distance in column $(L)$;

$\alpha=$ empirical coefficient accounting for solvent-sorbent interactions;

$\beta=$ empirical coefficient accounting for solvent-cosolvent interactions;

$\theta=$ volumetric liquid content of the soil $\left(L^{3} / L^{3}\right)$;

$\rho=$ dry bulk density $\left(M / L^{3}\right)$;

$\sigma=$ cosolvency power; and

$\omega=$ Damkohler number.

\section{Subscripts}

$m=$ mixed solvents; and

$w=$ water solvent. 